\title{
AB INITIO STUDY OF THE POSSIBLE SINGLE-CENTER UNITS FOR BINUCLEAR IRON COMPLEX $\left[\mathrm{Fe}_{2}(\mathrm{bpym})_{3} \mathrm{Cl}_{4}\right]$
}

\author{
Tihonovschi Andrei \\ Institute of Chemistry, Academy of Sciences of Moldova
}

\begin{abstract}
In present work we study two possible single-center units for binuclear iron complex $\mathrm{Fe}_{2}(\mathrm{bpym})_{3} \mathrm{Cl}_{4}-$ $\left[\mathrm{Fe}(\text { bpym })_{3}\right]^{2+}$ and $\mathrm{Fe}(\text { bpym })_{2} \mathrm{Cl}_{2}$. The obtained ground states for both studied systems are singlet states. In the case of $\mathrm{Fe}(\text { bpym })_{2} \mathrm{Cl}_{2}$ the lowest excited states were calculated to be $240 \mathrm{~cm}^{-1}$ (triplet) and $660 \mathrm{~cm}^{-1}$ (quintet) above the ground state and so are placed according to Lande rule. These states could be populated at room temperatures. For $\left[\mathrm{Fe}(\mathrm{bpym})_{3}\right]^{2+}$ first excited state was found to be about $6000 \mathrm{~cm}^{-1}$ above the ground state and so cannot be populated at normal temperatures.
\end{abstract}

Keywords: iron complexes, bipyrimidine complexes, magnetic properties, ab initio study.

\section{Introduction}

The binuclear and polynuclear transition metal complexes are widely used as models of natural enzymatic systems as well as models for molecular magnets. These complexes could be paramagnetic or diamagnetic in dependence of the bridging ligand(s) and ligand environment, manifesting the ferromagnetic or antiferromagnetic interactions between localized metal spins, respectively.

In order to explain magnetic properties of such complexes the Heisenberg-Dirac-Van Vleck model is widely used (see, for instance [1,2]). This model takes into account so-called localized spin values. These are nothing else than values supposed according to the electronic configurations of the metal centers and ligand field. These assumptions make sense when they give good fit to experimental data and there are no possibilities to measure experimentally or calculate theoretically the exact spin values on centers. At the same time these presumptions can give only very general understanding of the way interactions take place by. The exact on-center spin values (measured or calculated) can give a much better comprehension about mechanism of these processes.

Considering the binuclear system mentioned above $\left(\left[\mathrm{Fe}_{2}(\mathrm{bpym})_{3} \mathrm{Cl}_{4}\right]\right.$, see Fig. 1) we can choose one center and nearest ligands to form a single-center monomeric unit (marked with circle in Fig.1)

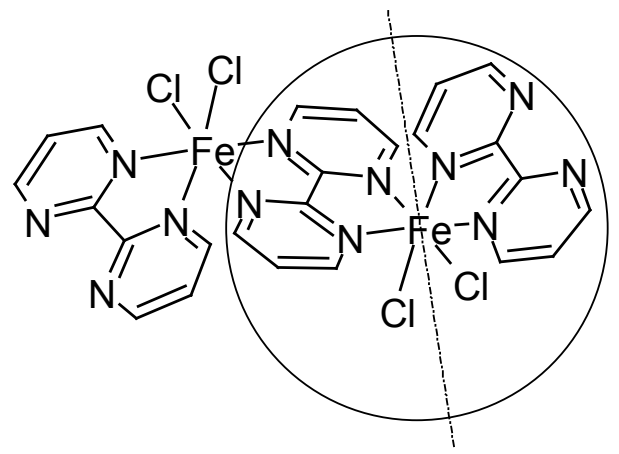

Fig. 1. $\mathrm{Fe}_{2}(\text { bpym })_{3} \mathrm{Cl}_{4}$ binuclear complex. Monomeric unit $\mathrm{Fe}(\text { bpym })_{2} \mathrm{Cl}_{2}$ is marked with a circle around, dotted line shows the $\mathrm{C}_{2}$ axis.

This unit seems to be symmetrical. The highest symmetry group possible in this case is $\mathrm{C}_{2}$. The main symmetry axis is also shown in Fig.1.

The monomeric unit shown before was selected as first possible monomer. The second monomer considered is $\left[\mathrm{Fe}(\mathrm{bpym})_{3}\right]^{2+}$ complex. It is well-known compound with similar ligand environment (only two chlorine atoms are substituted by bipyrimidine molecule). The magnetic properties of this monomer are described in experimental works $[3,4]$. Both monomeric units are shown on Fig.2. Because of the similarity of ligand environment the metal atoms in binuclear compound most likely have the same electronic configuration (and magnetic characteristics) as in these singlecenter molecules. 


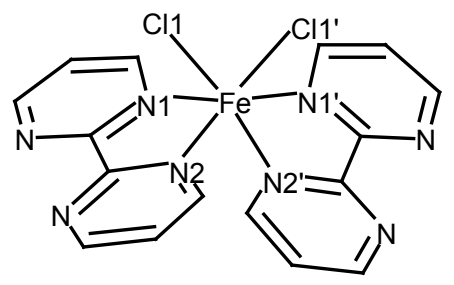

(1)

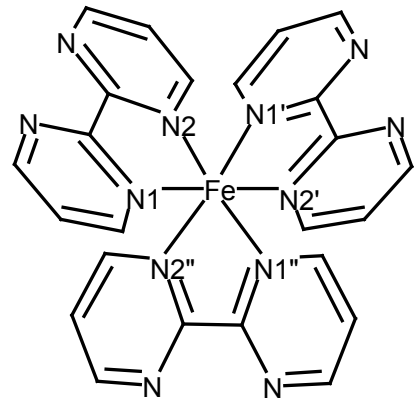

(2)

Fig. 2. $\mathrm{Fe}(\text { bpym })_{2} \mathrm{Cl}_{2}(1)$ and $\left[\mathrm{Fe}(\text { bpym })_{3}\right]^{2+}(2)$ systems with atom numbering.

\section{Methods of calculations}

Geometry configurations of the systems under consideration were optimized using ab initio (ROHF) gradient methods realized in PC GAMESS [5] quantum chemistry package. Geometry optimizations were performed for states with total spin value (S) equal to 0 and 1.

Electronic structure calculations were performed using ROHF method with and without taking in consideration configuration interactions (CI). CI basis is discussed below.

In all calculations STO-6G (Slater-Type Orbital approximated by 6 Gauss-type orbitals) orbital basis set was used.

\section{Results and discussion}

The first studied compound is $\mathrm{Fe}(\mathrm{bpym})_{2} \mathrm{Cl}_{2}$ neutral complex with $\mathrm{C}_{2}$ symmetry as it was mentioned before. The second compound is the $\left[\mathrm{Fe}(\mathrm{bpym})_{3}\right]^{2+}$ complex. The highest possible point group for this case is $\mathrm{D}_{3}$ (see Fig.2). Three axes $\mathrm{C}_{2}$ can be selected to go through each one of three bipyrimidine molecules centers and iron atom and $\mathrm{C}_{3}$ axis could be selected to go through iron atom and to be perpendicular to the plane in which three $\mathrm{C}_{2}$ axes lay (in Fig. $2 \mathrm{C}_{3}$ axis is perpendicular to the image plane).

The geometry data on these complexes are given in Table.1. and Table.2. There are presented calculated geometry data for both complexes and experimental data for $\left[\mathrm{Fe}(\mathrm{bpym})_{3}\right]^{2+}$ reported by de Munno et al in [3] and van Albada G.A. et al in [4].

Table 1

Bond distances and angles in $\mathrm{Fe}(\mathrm{bpym})_{2} \mathrm{Cl}_{2}(1)$

\begin{tabular}{l|ccc|ccc|cccc}
\hline \multirow{2}{*}{} & \multicolumn{3}{|c|}{$\mathrm{R}(\mathrm{Fe}-\mathrm{A}), \AA$} & \multicolumn{3}{c}{$\angle(\mathrm{N} 1-\mathrm{Fe}-\mathrm{A}), \mathrm{deg}$} & \multicolumn{4}{c}{$\angle(\mathrm{Cl} 1-\mathrm{Fe}-\mathrm{A}), \operatorname{deg}$} \\
$\mathrm{A}$ & $\mathrm{N} 1$ & $\mathrm{~N} 2$ & $\mathrm{C} 11$ & $\mathrm{~N} 2$ & $\mathrm{~N} 1$ & $\mathrm{~N} 2$ & $\mathrm{C} 11$ & $\mathrm{~N} 1$ & $\mathrm{~N} 2$ & $\mathrm{~N} 2$ \\
\hline Calculated & 1.892 & 1.876 & 2.196 & 83.72 & 174,96 & 92.64 & 93.61 & 89.96 & 89.21 & 176.04 \\
\hline
\end{tabular}

Table 2

Bond distances and angles in $\left[\mathrm{Fe}(\mathrm{bpym})_{3}\right]^{2+}(2)$

\begin{tabular}{ccccc}
\hline & $\mathrm{R}(\mathrm{Fe}-\mathrm{N} 1), \AA$ & $\angle(\mathrm{N} 1-\mathrm{Fe}-\mathrm{N} 2), \mathrm{deg}$ & $\angle\left(\mathrm{N} 1-\mathrm{Fe}-\mathrm{N} 1{ }^{\prime}\right), \mathrm{deg}$ & $\angle\left(\mathrm{N} 1-\mathrm{Fe}-\mathrm{N} 2{ }^{\prime}\right), \mathrm{deg}$ \\
\hline Exp [3], [4] & $1.970,1.968-1.975$ & $81.0,81.04-81.66$ & $88.31-95.76$ & $173.90-175.41$ \\
Calculated & 2.043 & 80.16 & 91.05 & 172.60 \\
\hline
\end{tabular}


From tables data it is seen that calculated geometry (at least for complex 2) is in good accordance with experimental data.

Calculated one-electron states which were used for CI calculations are presented on Fig.2. Their analytical forms are given in Table 3 and Table 4. It is seen that in both cases many active orbitals include large contributions from iron d-orbitals.

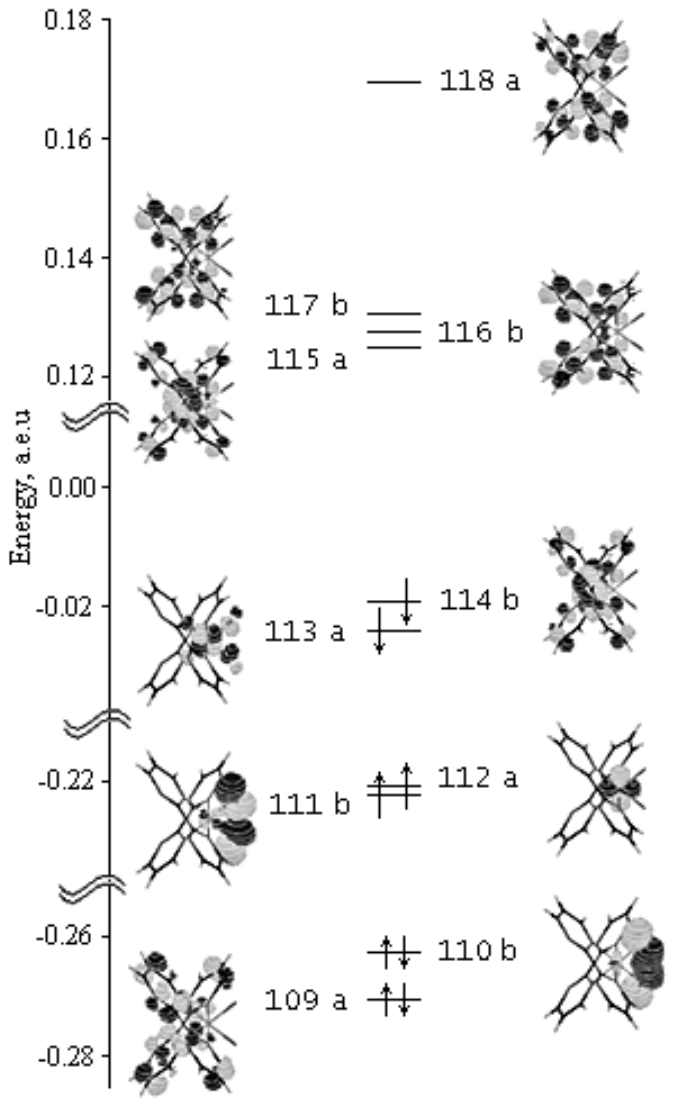

(1)

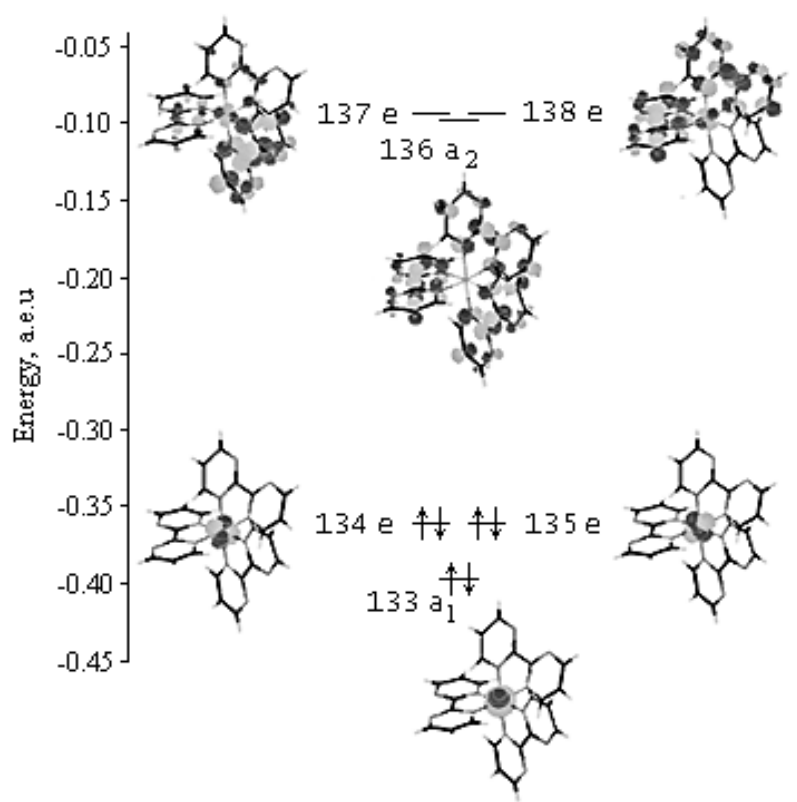

(2)

Fig.3. One-electron states of monomers $\mathrm{Fe}(\mathrm{bpym})_{2} \mathrm{Cl}_{2}(1)$ and $\mathrm{Fe}(\mathrm{bpym})_{3}{ }^{2+}(2)$. Electrons are placed according to the ground states obtained.

Table 3

MO LCAO for complex 1

\begin{tabular}{|c|c|}
\hline Orbital No, Sym & Composition \\
\hline $118 \mathrm{a}$ & $0.13 d_{x^{2}-y^{2}}^{F \theta}+0.23 d_{z^{2}}^{F \theta}+0.24 d_{x y}^{F \theta}+0.17 p^{\text {oipym }}$ \\
\hline $117 \mathrm{~b}$ & $0.18 p^{\text {Dipym }}$ \\
\hline $116 \mathrm{~b}$ & $0.18 p^{\text {bipym }}$ \\
\hline $115 \mathrm{a}$ & $0.17 p^{\text {blpym }} p^{\text {otpym }}$ \\
\hline $114 \mathrm{~b}$ & $0.29 d_{x^{2}-y^{2}}^{F \theta}+0.5 d_{z^{2}}^{F \theta}+0.52 d_{x y}^{F \theta}+0.1 p^{0 . p y m}$ \\
\hline $113 \mathrm{a}$ & $0.23 p^{\text {bipym }}$ (LUMO) \\
\hline 112 a & $\begin{array}{l}0.17 d_{x^{2}-y^{2}}^{r e}+0.29 d_{z^{2}}^{r e}+0.31 d_{x y}^{r e}+0.18 p^{b t p y m} \\
\left(\text { HOMO") }^{\text {ong }}\right.\end{array}$ \\
\hline $111 \mathrm{~b}$ & $0.5 p^{C l}$ \\
\hline $110 \mathrm{~b}$ & $0.45 p^{c l}$ \\
\hline 109 a & $0.68 p^{C l}$ \\
\hline
\end{tabular}




\begin{tabular}{|c|c|}
\hline & MO LCAO for complex 2 \\
\hline \begin{tabular}{|c|} 
Orbital No., \\
Sym
\end{tabular} & Composition \\
\hline $138 \mathrm{e}$ & $-0.20 d_{x^{2}-y^{2}}^{F \theta}-0.14 d_{y z}^{F \theta}+0.15 p^{i t g}$ \\
\hline $137 \mathrm{e}$ & $0.19 d_{x y}^{F \theta}+0.14 d_{x z}^{F \theta}+0.15 p^{\text {IIg }}$ \\
\hline $136 \mathrm{a}^{2}$ & $0.15 p^{\text {thg }} 0.15 p^{\text {tgg }}$ (LUMO) \\
\hline $135 \mathrm{e}$ & $0.78 d_{x^{2}-y^{2}}^{r e}+0.54 d_{y z}^{r e} 0.78 d_{x^{2}-y^{2}}^{r e}+0.54 d_{y z}^{r e}\left(\right.$ HOMO $\left.^{*}\right)$ \\
\hline $134 \mathrm{e}$ & $0.79 d_{x y}^{r e}+0.54 d_{x z}^{r e}$ \\
\hline $133_{\mathrm{a}} 1$ & $0.98 d_{z^{2}}^{F e}$ \\
\hline
\end{tabular}

The main criteria for choosing the MOs for CI basis were their composition (main contributing AOs) and the energy gap between them and HOMO (LUMO) level. The size of the CI basis was also chosen so that the possible number of configurations to be big enough.

For complex 1 it is seen that HOMO levels are two degenerated states with main contribution of $\mathrm{d}^{\mathrm{Fe}}$ orbitals as well as HOMO-1 level. LUMO is composed from p-orbitals of the three bipyrimidine ligands. HOMO and LUMO levels are very well separated $(\Delta \mathrm{E} \approx 6.8 \mathrm{eV}(0,25 \mathrm{a}$. u. $))$

In the case of the complex 2 the main contributors to the most active levels are d-orbitals of the iron atom, p-orbitals of the chlorine atoms and p-orbitals of the nitrogen atoms of the bipyrimidine ligand.

The CI calculations for $\mathrm{Fe}(\text { bpym })_{2} \mathrm{Cl}_{2}$ were performed using 108 core orbitals and 10 active with 8 electrons which give 22100 determinants with $\mathrm{S}_{\mathrm{z}}=0$. In case of the $\mathrm{Fe}(\text { bpym })_{3}{ }^{2+}$ we have used 132 core orbitals and 6 active with 6 electrons which give 400 determinants with $S_{z}=0$ ( 175 with $S=0 ; 189$ with $S=1 ; 35$ with $S=2$ and 1 with $S=3$ ).

The active orbitals in both cases were selected to include highest occupied levels that contain contributions from metal d-orbitals and so these orbitals can be considered "magnetic orbitals" (see [1]).

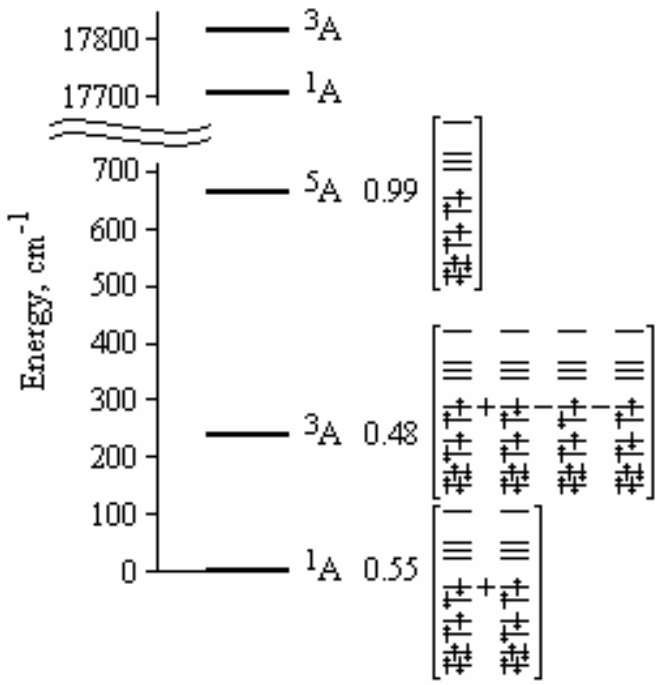

(1)

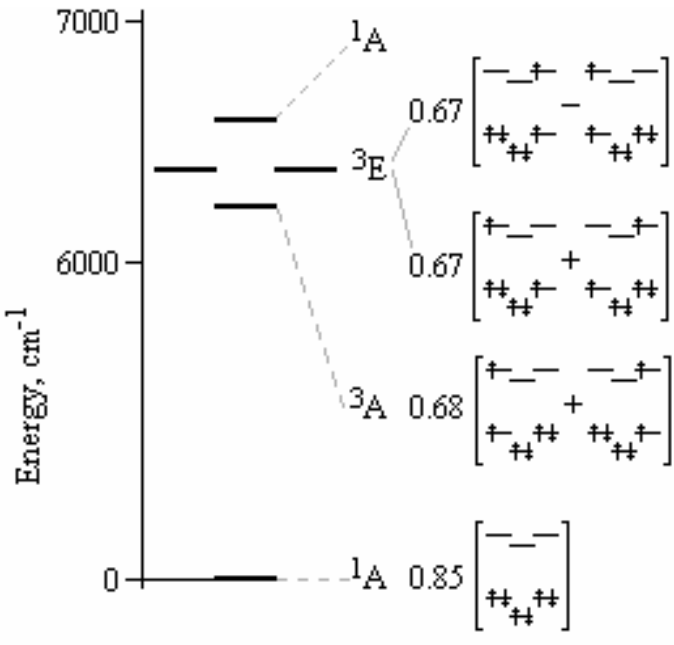

(2)

Fig.4. Lowest multielectronic states of the (1) $\mathrm{Fe}(\mathrm{bpym})_{2} \mathrm{Cl}_{2}$ and (2) $\left[\mathrm{Fe}(\mathrm{bpym})_{3}\right]^{2+}$. Only the most contributing electronic configurations are presented with corresponding coefficients. Orbitals populations are shown by arrows. 
The multielectronic states of the complexes 1 and 2 are presented on Fig. 4 . In case of the complex 1 we have very interesting picture. The levels are placed according to Lande rule. In this case the ground state is singlet. The first excited state which is triplet-one is only $240 \mathrm{~cm}^{-1}$ above the ground state and the second excited state is a quintet at $660 \mathrm{~cm}^{-1}$ above the ground state. In this case the lowest excited states could be populated at normal temperatures.

In case of complex 2 ground state is singlet too. This result is in accordance with experimental data given both in [3] and [4]. The gap between the ground and the first excited state is about $6100 \mathrm{~cm}^{-1}$ and so the excited states are not populated at normal temperatures.

\section{Conclusions}

The results obtained show that values of the localized spins on centers in $\mathrm{Fe}_{2}(\text { bpym })_{3} \mathrm{Cl}_{4}$ complex cannot be presupposed to be equal to 2 . The both studied single-center units were shown to have singlet ground states in accordance with experimental data and cannot be used in Heisenberg model. This means, that the only way to successfully develop any suitable model that will describe not only the behavior of the complete system, but the internal interactions that take place there, is to proceed with the magnetic properties of polynuclear complexes quantitatively by both the experimental or theoretical ab initio mean.

\section{References}

[1] Kahn O. Molecular magnetism. N.Y. : VCH Publishers Inc., 1993.

[2] Tsukerblat B.S. Group Theory in Chemistry and Spectroscopy. A Simple Guide to advanced Usage. London : Academic Press, 1994.

[3] De Munno G., Julve M., Real J.A. Inorg. Chim. Acta. 1997, Vol. 255, 1, pp. 185-188.

[4] van Albada G.A., Smeets W.J.J., Spek A.L. and Jan Reedijk. J. Chem. Cryst. 2000, Vol. 30, 7, pp. 441-444.

[5] Granovsky A. PC GAMESS version 7.0. http://classic.chem.msu.su/gran/gamess/index.html. 